\title{
Dynamical response of the perfect conducting channel in carbon nanotubes
}

\author{
Yoichi Asada and Tsuneya Ando \\ Department of Physics, Tokyo Institute of Technology, 2-12-1 Ookayama, Meguro-ku, Tokyo 152-8551, Japan
}

\begin{abstract}
The dynamical conductivity in metallic carbon nanotubes with impurities is studied based on an exact numerical diagonalization. The results show clearly that the conductivity deviates considerably from the usual Drude behavior due to the absence of backward scattering and the presence of a perfect conducting channel in the low-frequency region.
\end{abstract}

Keywords: carbon nanotube, effective-mass theory, dynamical conductivity, symplectic symmetry

PACS: $73.63 . \mathrm{Fg}, 78.67 . \mathrm{Ch}$

The electronic transport in carbon nanotubes has been a subject of extensive experimental and theoretical studies [1]. In metallic nanotubes, the backward scattering is absent even in the presence of scatterers unless their potential range is smaller than a few angstroms [2, 3] and there is a perfect conducting channel when there are several bands present at the Fermi level [4]. This unique property can be ascribed to the symplectic symmetry of the effective Hamiltonian in the $\mathbf{k} \cdot \mathbf{p}$ scheme and the presence of odd channel numbers [1, 4]. The dynamical conductivity was calculated in the self-consistent Born approximation (SCBA) [5]. The weak localization correction was calculated also [6]. In this paper, we calculate the dynamical conductivity based on an exact numerical diagonalization in order to clarify the importance of the perfect conducting channel.

We consider a metallic carbon nanotube with circumference $L$ and length $A(A \gg L)$. The metallic nanotube has conduction and valence bands crossing at the points corresponding to the $\mathrm{K}$ and $\mathrm{K}^{\prime}$ points of the Brillouin zone in the two-dimensional graphite. In the effective mass approximation, electronic states near the $\mathrm{K}$ point (or $\mathrm{K}^{\prime}$ point) are described by the $\mathbf{k} \cdot \mathbf{p}$ equation $[1,7]$. We consider impurities whose range of scattering potential is larger than the lattice constant but shorter than the electron wave length, typically $2 \pi / L$. Most of scatterers present in actual nanotubes are expected to satisfy this condition. For such scatterers, we can neglect the scattering between $\mathrm{K}$ and $\mathrm{K}^{\prime}$ points and these two points can be treated independently [2].

The Hamiltonian for the $\mathrm{K}$ point is given by

$$
H=\gamma(\vec{\sigma} \cdot \hat{\mathbf{k}})+\sum_{i} u_{i} \delta\left(\mathbf{r}-\mathbf{r}_{i}\right)
$$

Here $\gamma$ is a band parameter, $\hat{\mathbf{k}}=\left(\hat{k}_{x}, \hat{k}_{y}\right)$ the wave-vector operator defined by $\hat{\mathbf{k}}=-\mathrm{i} \vec{\nabla}+e \mathbf{A} / c \hbar$ with $\mathbf{A}$ being a vector potential, $\vec{\sigma}=\left(\sigma_{x}, \sigma_{y}\right)$ the Pauli spin matrices (two sites in a unit cell play a role of quasi-spin). We suppose that a magnetic flux $\phi_{x}$ is passing through the cross section of the nanotube. The positions $\mathbf{r}_{i}$ of impurities are distributed randomly and independently in the system and we assume equal amount of attractive and repulsive scatterers, $u_{i}= \pm u$. The strength of disorder is characterized by the dimensionless parameter $W=n_{\mathrm{i}} u^{2} / 4 \pi \gamma^{2}$ [5], with $n_{\mathrm{i}}$ being the impurity density. We impose periodic boundary conditions in the axis direction. For the purpose of numerical calculations, we restrict the basis by a cutoff energy $\varepsilon_{\mathrm{c}}=3(2 \pi \gamma / L)$. We use $|\sigma, \mathbf{k}\rangle(\sigma=\uparrow, \downarrow$ and $\left.\gamma|\mathbf{k}|<\varepsilon_{\mathrm{c}}\right)$ as a basis.

The number of conducting channels at the Fermi energy $N_{\mathrm{c}}$ can be varied without breaking symplectic symmetry by controlling the Fermi energy and the magnetic flux. When $\phi_{x}$ is an integer multiple of the flux quantum $\phi_{0}=c h / e, N_{\mathrm{c}}$ is odd irrespective of the value of the Fermi energy. When $\phi_{x}=\phi_{0} / 2, N_{\mathrm{c}}$ becomes even, for which the perfect channel disappears and states are localized in spite of the symplectic symmetry [8].

We use the Kubo formula to calculate the dynamical conductivity in the tube axis direction ( $y$-direction),

$$
\begin{aligned}
\sigma_{y y}(\omega)= & \frac{\pi \hbar}{L A}\left\langle\sum_{n, m}\left[f\left(\varepsilon_{m}\right)-f\left(\varepsilon_{n}\right)\right]\right. \\
& \left.\times \frac{\left|\left\langle n\left|\hat{j}_{y}\right| m\right\rangle\right|^{2}}{\varepsilon_{n}-\varepsilon_{m}} \delta\left(\hbar \omega-\varepsilon_{n}+\varepsilon_{m}\right)\right\rangle .
\end{aligned}
$$

Here, $f(\varepsilon)$ is the Fermi distribution function, $\varepsilon_{n}$ and $|n\rangle$ represent $n$th eigenvalue and eigenvector, respectively, $\hat{j_{y}}=-(e \gamma / \hbar) \sigma_{y}$ is the current operator, and $\langle\cdots\rangle$ means the sample average. In practice, we calculate the conductivity averaged in a small frequency window $[\omega-$ $\left.\delta_{\omega} / 2, \omega+\delta_{\omega} / 2\right]$ to avoid the delta function in the Kubo formula, and the frequency dependence of the conductivity is shown like a histogram.

We present numerical results for $N_{\mathrm{c}}=1$ and 3 . It is known that the backward scattering is absent in the case 


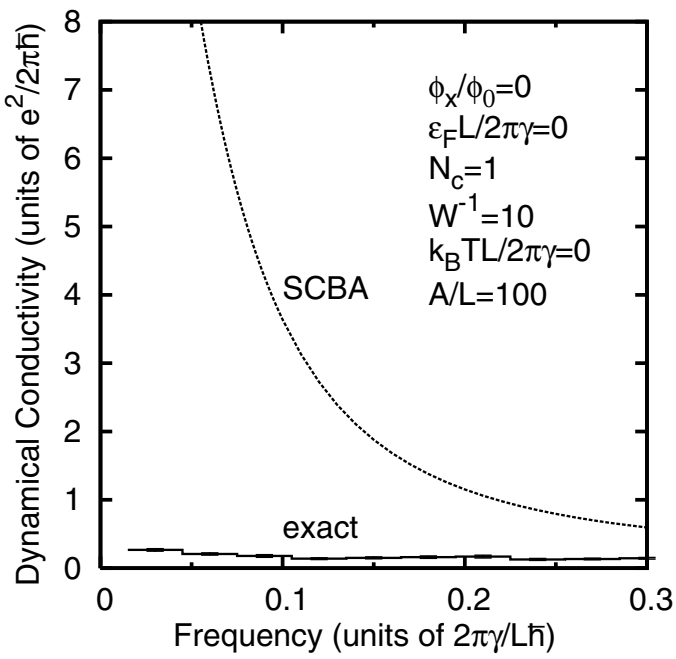

FIGURE 1. Dynamical conductivity for $A / L=100, W^{-1}=$ $10, k_{\mathrm{B}} T L / 2 \pi \gamma=0, \phi_{x} / \phi_{0}=0, \varepsilon_{F} L / 2 \pi \gamma=0$, and $N_{c}=1$ obtained in the SCBA and by exact numerical diagonalization.

of $N_{\mathrm{c}}=1$, i.e., the transmission is perfect [2]. In the case of $N_{\mathrm{c}}=3$, there is one perfect conducting channel and the conductance of a finite-length nanotube decreases from the ideal value corresponding to $N_{\mathrm{c}}=3$ to that in the single channel case as the length of nanotube increases [4]. The similar conclusions can be obtained in different methods [9].

A numerical result of the dynamical conductivity for $N_{\mathrm{c}}=1$ in a small-frequency region is shown in Fig. 1 . The result indicates that the Drude behavior cannot be seen in contrast to that in SCBA. It is likely that the conductivity in the vicinity of $\omega=0$ is not broadened and there is a delta function at zero frequency.

Figure 2 shows a result for $N_{\mathrm{c}}=3$. When the frequency is larger than $\omega_{\mathrm{c}} \approx 0.1 \times(2 \pi \gamma / L \hbar)$, the dynamical conductivity obtained by exact diagonalization is nearly the same as that in SCBA, i.e., the Drude behavior is observed. On the other hand, in a small frequency region, the exact dynamical conductivity deviates from that in the SCBA and decreases with the decrease of the frequency.

The numerical result indicates that a crossover occurs at $\omega_{\mathrm{c}} \approx 0.1 \times(2 \pi \gamma / L \hbar)$ in the case of $\phi_{x} / \phi_{0}=0$, $\varepsilon_{\mathrm{F}} L / 2 \pi \gamma=1.5, N_{\mathrm{c}}=3$, and $W^{-1}=10$. This corresponds to the crossover from the Drude transport regime to the "single-channel" transport regime. The result suggests again that there is a delta-function conductivity at zero frequency even in the case of $N_{\mathrm{c}}=3$ related to the perfect conducting channel.

More detailed results and discussions, including the case of $N_{\mathrm{c}}=2$ where the Anderson localization takes place, are reported elsewhere [10]. Further, a detailed study on the presence of a delta function at zero fre-

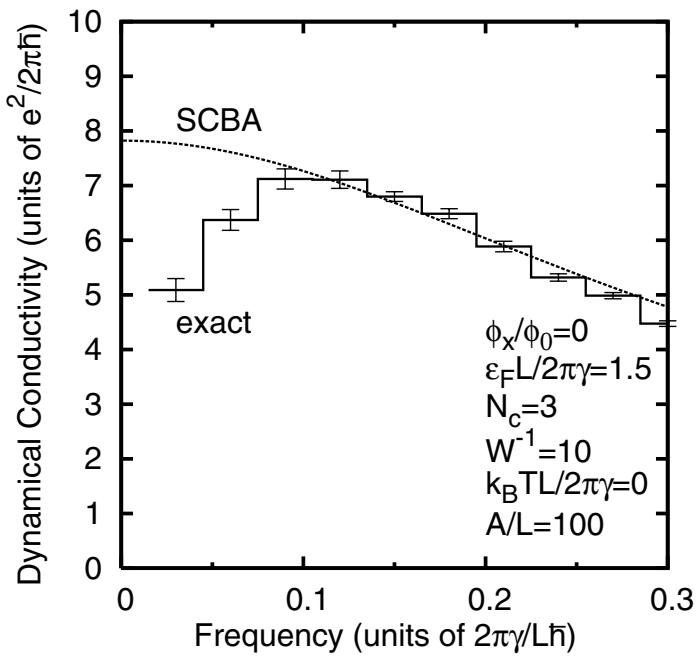

FIGURE 2. Dynamical conductivity for $A / L=100, W^{-1}=$ $10, k_{\mathrm{B}} T L / 2 \pi \gamma=0, \phi_{x} / \phi_{0}=0, \varepsilon_{F} L / 2 \pi \gamma=1.5$, and $N_{c}=3$ obtained in the SCBA and by exact numerical diagonalization.

quency is desirable but left for the future.

\section{ACKNOWLEDGMENTS}

One of the authors (Y.A.) acknowledges the support of Research Fellowships of the Japan Society for the Promotion of Science for Young Scientists. This work was supported in part by a 21 st Century COE Program at Tokyo Tech "Nanometer-Scale Quantum Physics" and by Grant-in-Aid for Scientific Research from the Ministry of Education, Culture, Sports, Science and Technology, Japan.

\section{REFERENCES}

1. T. Ando, J. Phys. Soc. Jpn. 74, 777 (2005).

2. T. Ando and T. Nakanishi, J. Phys. Soc. Jpn. 67, 1704 (1998).

3. T. Ando, T. Nakanishi, and R. Saito, J. Phys. Soc. Jpn. 67, 2857 (1998).

4. T. Ando and H. Suzuura, J. Phys. Soc. Jpn. 71, 2753 (2002).

5. T. Ando, J. Phys. Soc. Jpn. 71, 2505 (2002).

6. H. Suzuura and T. Ando, J. Phys. Soc. Jpn. 75, 024703 (2006).

7. H. Ajiki and T. Ando, J. Phys. Soc. Jpn. 62, 1255 (1993).

8. T. Ando, J. Phys. Soc. Jpn. 75, 054701 (2006).

9. Y. Takane and K. Wakabayashi, J. Phys. Soc. Jpn. 72, 2710 (2003). Y. Takane, J. Phys. Soc. Jpn. 73, 9 (2004); 73, 1430 (2004); 73, 2366 (2004).

10. Y. Asada and T. Ando, J. Phys. Soc. Jpn. (submitted for publication). 